\title{
Nursing Intervention of Aspiration Complicated by Enteral Nutrition in Elderly Patients
}

\author{
Luting Chen \\ Nanjing General Hospital, Nanjing, 210002, China
}

Keywords: Elderly patients, Aspiration, Enteral nutrition.

\begin{abstract}
Objective To investigate nursing measures for enteral nutrition complicated aspiration in elderly patients and countermeasures to prevent aspiration. Methods Select 68 elderly patients who received enteral nutrition therapy in our hospital from January 2016 to January 2017. The patients were divided into control group and observation group. The number of patients in each group was 34 . The control group was treated with routine nursing method. And the observation group was treated with quality nursing management. The incidence of aspiration and aspiration pneumonia was observed and compared. Results The incidence of aspiration (2.94\%) and the incidence of aspiration pneumonia ( $0 \%$ ) of the observation group were significantly lower than that of the control group (the incidence of aspiration and aspiration pneumonia were $29.41 \%$ and $20.59 \%$, respectively), statistically significant $(\mathrm{P}<0.05)$. Conclusions The clinical effect of quality nursing management intervention for elderly patients with enteral nutrition is better, and it can effectively prevent the occurrence of aspiration. At the same time, after the occurrence of aspiration, immediately take the appropriate care measures, aspiration pneumonia can be effectively prevented.
\end{abstract}

\section{Introduction}

Enteral nutrition refers to the nutrition support way the gastrointestinal tract provides for patients for metabolic required nutrients, usually used in patients with disturbance of consciousness, dysphagia or severe gastrointestinal diseases. The clinical application of enteral nutrition support is better, but in the operation, aspiration may be caused by improper operation or other causes, resulting in patients with aspiration pneumonia. Especially for elderly patients, the concurrent aspiration will significantly improve the mortality rate of patients. Therefore, it is very positive to strengthen the nursing management for elderly patients for the prevention of aspiration during therapy. In this study, take 68 elderly patients who received enteral nutrition therapy in our hospital from January 2016 to January 2017 as observation objects to observe the clinical effect of quality nursing management intervention for preventing aspiration in elderly patients and to explore the relevant nursing measures, as reported below.

\section{Data and method}

\section{General data}

Select 68 elderly patients who received enteral nutrition therapy in our hospital from January 2016 to January 2017. The patients were divided into control group and observation group. The number of patients in each group was 34. The number of male and female patients in the control group was 21 and 13. The minimum age and the maximum age was 81 and 96, and the average age was (87.06 \pm 2.84); there were 11 patients with consciousness disorder, 12 patients with dysphagia, and 11 patients with severe gastrointestinal diseases. In the observation group, the number of male and female patients was 20 and 14. The minimum age and the maximum age was 82 and 95, and the average age was (87.51 \pm 2.43$)$; there were 11 patients with consciousness disorder, 11 patients with dysphagia, 
and 12 patients with severe gastrointestinal diseases. There was no significant difference between the general data of these two groups, and the data comparison was not statistically significant $(\mathrm{P}>0.05)$.

Exclusion criteria: (1) death after therapy; (2) contraindications; (3) patients who do not cooperate.

\section{Nursing method}

The control group was treated with routine nursing method, including the provision of nutrient solution for patients, helping patients to complete the input of the nutrient solution, ensuring the catheterizing position clean, and doing the relevant nasal lubrication work. In addition to the above measures, the observation group received quality nursing management intervention, and the specific method is as follows.

Training of nursing staff

Train nursing staff responsible for enteral nutrition, strengthen their understanding of enteral nutrition, and improve the theoretical awareness and related operational skills of nursing process, aspiration prevention measures and emergency response to the occurrence of aspiration, so as to fully improve the professional level of nursing staff. Through the theoretical examination, on-site operation, scenario simulation, etc. conduct assessment for nursing staff, and only nursing staff who pass the assessment can participate in nursing work of patients in the observation group.

\section{Evaluation of patient condition}

Before the implementation of enteral nutrition support for older elderly patients, the nursing staff should conduct a comprehensive assessment on the patients' overall condition, such as disease progression, swallowing function, gastrointestinal function, cognitive function, and gastric remnant, understand whether the patient has contraindications, and carry out nursing work according to the basic situation of patients. Swallowing reflexes of elderly patients with consciousness disorder is relatively weak, and the probability of aspiration during enteral nutrition support is greater, therefore, for older patients with conscious disability, especially obnubilation or Glasgow Coma Scorecard (GCS) score $<9$ points, before nasal feeding, turn over, and exhaust the respiratory tract secretions, to avoid the aspiration caused by saliva, blood and gastrointestinal reflux liquid.

Nursing of patient position

In case of enteral nutrition for elderly patients, the choice of the patients' position is very important. If the position is not appropriate, it is very easy to lead to aspiration. Therefore, in the event of implementation of enteral nutrition support operation, choose the right position for patients. Nursing staff can appropriately raise the bed by $30{ }^{\circ}$ to $40^{\circ}$, so that keep $30^{\circ}$ to $40^{\circ}$ lying position of patients in the nasal feeding process and an hour after the completion of nasal feeding.

Nursing of nutrition solution infusion

In case of nasal feeding for elderly patients, based on the actual condition of patients, choose the appropriate model of gastric tube, and insertion depth. When the diameter of the gastric tube is too large, the patient's lower esophageal sphincter may expanse, causing reflux and aspiration of gastrointestinal fluid. Selection of the appropriate gastric tube can reduce the probability of aspiration of patients. At the same time, before nursing staff conduct the intestine nutrition support operation, first monitor the residual amount in the stomach of patients, thereby reducing the incidence of aspiration. Nursing staff should every 4 hours conduct the gastric residual determination for patients. In case of too much residual in stomach, should suspend the infusion of nutrient solution and use gastric motility for treatment, and continue the nutrient solution infusion until the residual amount keeps normal. In addition, nursing staff should also choose the infusion method based on different circumstances of elderly patients, including one-time gravity infusion, intermittent gravity infusion or nutritional pump infusion. For enteral nutrition for elderly patients with artificial airway, conduct aspiration of subglottic secretions, 1time / $4 \mathrm{~h}$.

Treatment measures for aspiration patients

For elderly patients, aspiration easily leads to the emergence of aspiration pneumonia. In case of aspiration, immediately stop the infusion of nutrient solution, and lower the bed head, so that patients take the right lateral position, timely suck out the inhalation in patients's airway and the stomach contents through tube feeding. While encourage patients to cough, and by gently tapping help patients 
cough up the aspiration content, or directly use the trachea to remove the inhalation. The above measures can reduce the probability of lung infection in patients, to avoid the occurrence of aspiration pneumonia.

\section{Observation indicators}

Observe the incidence of aspiration and aspiration pneumonia in both groups. Aspiration incidence $=$ number of patients with aspiration $/$ number of patients in this group $\times 100 \%$. Aspiration pneumonia incidence $=$ number of patients with aspiration pneumonia $/$ number of patients in this group $\times 100 \%$.

\section{Statistical method}

The data was analyzed by SPSS16.0 statistical software and the measurement data was expressed by ( $\bar{x} \pm s)$, and t was used for testing. The count data was expressed as percentage (\%), and $\mathrm{x}^{2}$ was used for testing. In case of $\mathrm{P}<0.05$, the difference was statistically significant.

\section{Results}

In the observation group, there was 1 case of aspiration, and the incidence was 0 ; 0 case of aspiration pneumonia, and the incidence $0 \%$. In the control group, there was 10 case of aspiration, and the incidence was $29.41 \%$; 7 cases of aspiration pneumonia, and the incidence $20.59 \%$. There was significant difference in the incidence of aspiration and aspiration pneumonia in these two groups ( $\mathrm{P}$ $<0.05)$.

\section{Discussion}

Enteral nutrition is a nutrition support method commonly used by patients with dysphagia, consciousness disorder, severe gastrointestinal disorder, which can provide patients with all kinds of nutrients needed for metabolism, thus maintaining the diet balance during treatment ${ }^{[1]}$. But aspiration occurs in many patients with clinical implementation of enteral nutrition. The liquid in the gastrointestinal tract is mistakenly inhaled into the throat and trachea, and into the patient's lung, leading to that patients have pulmonary infection or inhalation pneumonia, thereby increasing the difficulty of treatment and mortality ${ }^{[2]}$.

Most patients with aspirations are elderly patients, and the main pathogenic factors are the follows. (1) The physiological factor. The function of each organ of the elderly is significantly diminished, the nerve reflex function is gradually degraded, gastrointestinal motility is poor, and the stomach takes a long time to digest the food, prone to gastric retention. In case of the nutrient fluid infusion speed is too fast, aspiration may easily occur ${ }^{[3]}$. (2) The nasogastric tube factor. Enteral nutrition has a higher demand for the choice of nasogastric feeding tube model and operation method. If the nasogastric tube model does not match with the patient, or insertion depth or fixed position is not proper, aspiration may easily occur. (3) The position factor. In case of enteral nutrition support operation, many nurses do not attach importance to the choice of the patient position, such as bed head elevation angle is too high or too low, or do not tell the patient not to violently turn over during the nasal feeding process, resulting in the occurrence of aspiration ${ }^{[4]}$. (4) Unreasonable nutrient infusion. Some elderly patients have poor digestion function, and there is more gastrointestinal nutrition solution residue. If the gastrointestinal residue is not sucked out first, direct input of a fixed amount of nutrition solution will lead to emergence of aspiration ${ }^{[5]}$. It can be seen that aspiration complicated by enteral nutrition is largely due to improper nursing management methods by nursing staff, therefore, to strengthen the nursing management for patients with enteral nutrition support is very important ${ }^{[6]}$. Nursing operation can only reduce the probability of occurrence of aspiration to a certain extent, but cannot completely avoid the occurrence of aspiration. And in case of aspiration, the nursing staff should also take immediate emergency measures to avoid lung infection and induced aspiration pneumonia. 
In the routine nursing model, the nursing staff does not form the prevention consciousness of absorption complications, and does not pay enough attention to details of the enteral nutrition nursing work, leading to the high incidence of aspiration and the concurrency of aspiration pneumonia, and the patient's condition worsened ${ }^{[7]}$. In recent years, the nursing level in the hospital has been greatly improved, nursing staff service skills and service attitude have greatly changed, enteral nutrition nursing work gets more and more attention, and quality nursing management intervention has been applied to enteral nutrition support work for elderly patients, and achieved remarkable results [8]. The nursing method fully considers the situation of elderly patients, and before nursing such patients, nursing staff receives relevant training to improve the theoretical awareness and operational level, and will undergo the strict assessment. Those who pass the assessment can participate in nursing work [9]. During nursing, analyze the causes of aspiration of patients with enteral nutrition and take corresponding preventive measures. Nursing will be carried out from several aspects, such as disease monitoring, patient position and nutrition infusion process, to normalize nursing operations of enteral nutrition support, so as to enhance the nursing level, greatly reduce the probability of patients with aspiration [10]. At the same time, for elderly patients with aspiration, immediately take the appropriate treatment measures to avoid aspiration into the patient's lungs and resulting in infection, thereby reducing the probability of aspiration pneumonia. Compared with the routine nursing method, quality nursing management intervention is more meticulous, targeted and humane.

In this study, we selected 68 elderly patients who received enteral nutrition therapy in our hospital from January 2016 to January 2017 for observation, and respectively conducted routine nursing (as control group) and quality nursing management intervention (as observation group). The results showed that the incidence of aspiration and aspiration pneumonia in the observation group is significantly lower than those in the control group, and the difference was statistically significant ( $>$ 0.05). To sum up, quality nursing management intervention in elderly patients with enteral nutrition and aspiration has better application effect, and can reduce the incidence of aspiration and induced aspiration pneumonia, therefore, able to be promoted and used.

\section{References}

[1] Shao Li. Prognosis Effect of Enteral Nutrition Nursing Intervention on Patients with Acute Cerebral Hemorrhage, World Latest Medical Information Digest, 2017, 7 (28): 227, 230.

[2] Guo Suhua, Wu Xiaoting, Zhou Ronghua. Application Fine Nurse in Enteral Nutrition Management of Patients with Severe Traumatic Brain Injury, Qilu Nursing Journal, 2017,9 (12): 83-85.

[3] Zhong Shigui, Li Xiaomang, Yi Caixia, Hong Jing, Huang Li, Li Shenmei, Zhao Yumin. Effects of Liquid-capsule Jejunum Tube Enteral Nutrition on Postoperative Patients with Colorectal Cancer, Qilu Nursing Journal, 2017, 7 (12): 101-102.

[4] Chen Yu Mei. Clinical Significance of Early Enteral Nutrition of Patients with Craniocerebral Injury, Chinese and Foreign Medical, 2017, 8 (15): 29-31.

[5] Tan Xiaohui, She Lijun, Huang Peiming, Liang Hui. Role of Cluster Nursing in Preventing Aspiration of Patients with Severe Respiratory Syndrome, Journal of Chinese Contemporary Medicine, 2017, 12 (17): 194-196.

[6] He Xiaolan, Zhou Xueling. Application of Quality Control Circle Activities in Reducing the Incidence of Enteral Nutrition Aspiration, Health Vocational Education, 2017, 35 (09): 158-159.

[7] Li Junhua. Effect of Low calorie enteral nutrition nursing on intestinal mucosal barrier function and nutritional status of patients with severe cerebral infarction, Modern Medicine and Health, 2017, 7 (10): 1552-1553. 
[8] Wang Weili. Nurse Experience of Early Enteral Nutrition Support via Nose-intestine Nutritional Tube on Patients with Severe Acute Pancreatitis, Journal of Henan Surgery, 2017, 9 (03): 173-174.

[9] Shi Yanli. Clinical Nurse of Early Enteral Nutrition via Nasojejunal Tube after Pancreaticoduodenectomy, Chinese Medicine Guide, 2017, 22 (12): 239-240.

[10]Li Ping, Wen Jin, Nie Yuqin. Clinical Efficacy of Parenteral and Enteral Nutrition Sequential Therapy for Patients with Severe Mechanical Ventilation, China Medical Herald, 2017, 7 (14): 107-110. 